\title{
Cognitive behavioural therapy improved psychiatric symptoms in drug refractory schizophrenia
}

Sensky T, Turkington D, Kingdon D, et al. A randomized controlled trial of cognitive-behavioral therapy for persistent

symptoms in schizophrenia resistant to medication. Arch Gen Psychiatry 2000 Feb;57:165-72.
QUESTION: Is cognitive behavioural therapy (CBT) more effective than a befriending intervention for reducing psychiatric symptoms in drug refractory schizophrenia?

Design

Randomised (allocation concealed), blinded (outcome assessors), controlled trial with 9 months of follow up after treatment.

\section{Setting}

5 clinical services in the UK (Newcastle, Cleveland, Durham, and 2 in west London).

\section{Patients}

90 patients who were 16-60 years of age (mean age $39 \mathrm{y}$, $59 \%$ men, $89 \%$ white), met $I C D-10$ research and $D S M-I V$ criteria for schizophrenia, and had symptoms causing distress or dysfunction for $\geq 6$ months despite adequate doses of antipsychotic drugs. Follow up was $100 \%$ for all outcomes except for scores on the Scale for Assessment of Negative Symptoms (SANS), which had 98\% follow up.

\section{Intervention}

Patients were allocated to CBT $(\mathrm{n}=46)$ or befriending $(\mathrm{n}=44)$. Both interventions were done by the same experienced nurses. CBT involved attaining a collaborative understanding of the development of symptoms and working towards reducing distress and disability. The provision of CBT was guided by a manual and regular supervision. Befriending involved sessions focused on neutral topics (ie, hobbies, sports, and current affairs). Sessions were approximately 45 minutes/week for the first 2 months with reduced frequency for the next 7 months (mean total of 19 sessions). Patients in both groups were on antipsychotic medication throughout the study period.

\section{Main outcome measures}

Sources of funding: Wellcome Trust and Hounslow and Spelthorne Community and Mental Health

National Health Service Trust.

For correspondence: Dr T Sensky, Division of

Neurosciences and

Psychological Medicine,

Imperial College School

of Medicine, West

Middlesex University

Hospital, Isleworth,

Middlesex TW7 6AF

UK.t.skensky@ic.ac.uk with $\geq 50 \%$ improvement in CPRS schizophrenia change scores $(\mathrm{p}=0.06)$ (table). Groups did not differ for patients with $\geq 50 \%$ improvement in MADRS or SANS scores.

\section{Conclusion}

Cognitive behavioural therapy led to greater improvement in psychiatric symptoms at 9 months follow up than did a non-specific befriending intervention in patients with drug refractory schizophrenia.

\section{COMMENTARY}

A recent Cochrane review on the use of CBT for schizophrenia showed promising findings but recommended further large, methodologically rigorous trials. ${ }^{1}$ In their well designed and controlled study, Sensky et al compare the role of CBT and befriending for drug resistant schizophrenia. Although both groups showed improvements immediately after treatment, only the CBT group continued to improve after the treatment ended. Clinical improvements were greater for CBT than for befriending in all 4 outcome measures at 9 months of follow up. At the beginning of the study, patients showed no evidence of poorer adherence to medication, and by the end of the study, the results could not be accounted for by changes in antipsychotic medication.

Strengths of this study are randomised treatment allocation, blinded outcome assessors, audiotaped sessions to ensure that the nurses delivered the assigned intervention, medication monitoring for all patients, and an adequate follow up period accounting for all participants. Weaknesses are possible contamination by therapists conducting both interventions, insufficient detail about the severity of patient psychosis, inadequate social profiles (eg, socioeconomic status) of participants to determine representativeness of study patients to all those with drug resistant symptoms, and an absence of measures such as relapse and readmission rates.

The positive findings suggest greater internalised ability and skill acquisition by patients in the CBT group to control or cope with their persistent symptoms at follow up, whereas those in the befriending group lost their initial gains once direct contact with the therapist ended. Given that up to $50 \%$ of patients with schizophrenia are non-responsive to antipsychotic agents, effective psychological interventions, such as CBT, are viable alternatives or adjuncts to medication. The cost of specialised CBT training has not been accounted for in this investigation and is recommended for future research. It appears that CBT by trained and experienced therapists is effective for patients who are drug resistant and who do not experience severe symptoms that would interfere with the requirements of this intervention. Future research should more clearly describe those patients who may benefit.

Jeannette LeGris, RN, MHSc Clinical Nurse Specialist, Psychiatry Assistant Professor, School of Nursing McMaster University Hamilton, Ontario, Canada

1 Jones C, Cormac I, Mota J, et al. Cognitive behaviour therapy for schizophrenia (Cochrane Review, latest version 28 Jan 2000). In: Cochrane Library. Oxford: Update Software.

${ }^{*} \mathrm{CPRS}=$ Comprehensive Psychiatric Rating Scale. Other abbreviations defined in glossary; RBI, NNT, and $\mathrm{Cl}$ calculated from data in article. 